\title{
Electron diffraction studies of pulsed cluster beams
}

\author{
L.S. Bartell and R.J. French \\ Department of Chemistry, University of Michigan, Ann Arbor, Mr 48109, USA
}

Received 5 July 1988

A pulsed nozzle source has been installed in the electron diffraction unit at the University of Michigan. The pulsed mode of operation is found to offer several important advantages in the investigation of clusters generated in nozzle flow. These advantages, including the feasibility of operating without a skimmer, are discussed. Design features and characteristic results are briefly outlined.

PACS: $36.40 ; 47.55 . E ; 61.14 . F$

\section{Introduction}

Modifications of the electron diffraction unit at the University of Michigan have greatly facilitated research on molecular clusters generated by homogeneous nucleation in nozzle flow. Included are a pulsed nozzle and auxiliary equipment to characterize the supersonic jets produced. Advantages of pulsed operation are several. Briefly stated, sample pressures can be increased by an order of magnitude without alteration of the pumping system, and substantially lower temperatures can be attained. It is now possible to produce cluster beams of such volatile substances as $\mathrm{Ne}$ (at $10^{\circ} \mathrm{K}$ ) that previously resisted condensation in the Michigan apparatus. The increased range of expansion conditions markedly augments the control that can be exercised over the internal structure of clusters formed in nozzle flow. Moreover, the improved quality of the supersonic jets makes it possible under some conditions to obtain serviceable electron diffraction patterns of clusters without skimming the jets and thereby interfering with their flow characteristics.

Although spectroscopists have long taken advantage of the attractive features of pulsed jets, diffractionists have been slow to adopt such a system because of perceived problems associated with its use. It turns out that the problems need not be severe. A description of the pulsed system is given in the next section together with a discussion of measures taken to overcome the interferences encountered when a pulser operates in the vicinity of an electron beam.

\section{Description of apparatus}

The pulsed nozzle system consists of a commercial electromagnetic solenoid valve, designed for spectroscopic research, controlling the flow through a miniature Laval nozzle. A high-speed shutter actuating the $40 \mathrm{kev}$ electron beam can be timed to examine any desired interval of the gas pulses. A beam chopper and flight tube monitored by a fast ionization gauge can be introduced into the system to display the distribution of molecular and cluster velocities in a jet. In other aspects the Michigan electron diffraction unit is unchanged from its configuration described elsewhere [1]. Under some operating conditions, however, diffraction patterns are recorded with the skimmer removed. Such a mode of operation will be discussed later.

Of greatest concern in initial planning was the possibility that electromagnetic pulses associated with the triggering of the pulsed valve might severely disturb the electron beam and spoil diffraction patterns. This potential source of trouble has been overcome, partly by ensheathing the solenoid with three 
layers of magnetic shielding to reduce the deflection of the electron beam to a few hundredths of an $s$-unit. In addition, the unwanted magnetic pulse decays to a small fraction of its maximum value in the nearly $1 \mathrm{~ms}$ time it takes the jet to reach the electron beam after the initial surge of current through the solenoid. During the brief, reproducibly timed period that the electron beam is switched on to probe the jet, any deflection is so small and static that no degradation of the diffraction pattern has been detectable.

Contained in a unit less than $2.5 \mathrm{~cm}$ in diameter and $5 \mathrm{~cm}$ in length, the pulser valve and electromagnetic solenoid driving it are so compact that they could be introduced into the apparatus with only minor alterations. Materials to which gas samples are exposed besides the nozzle are teflon, viton, and stainless steel. The valve is rated for operating pressures up to 86 bars and is designed to produce pulse widths from $250 \mu \mathrm{s}$ FWHM to continuous with a $160 \mu$ s opening time, $350 \mu \mathrm{s}$ closing time, and a repetition rate exceeding $250 \mathrm{~Hz}$ for short pulses. A $300 \mathrm{~V}$ pulse, $150 \mu \mathrm{s}$ FWHM opens the valve followed by a sustained application of about $10 \mathrm{~V}$ to hold the valve open.

In the commonest configuration of the pulsed source a miniature glass Laval nozzle, throat diameter $\approx 0.012 \mathrm{~cm}$, is joined to the valve, being cushioned at both ends by teflon gaskets. The primary pressure seal is made by an O-ring around the nozzle near its throat. Between the nozzle and the valve is a dead space of about $10^{-3} \mathrm{~cm}^{3}$ whose half-life for emptying after the valve is closed is less than $1 \mathrm{~m} \mathrm{~s}$. At the repetition rate normally used in experiments the dead space is exhausted long before a new pulse begins.

Diffracted electrons are filtered, as usual, by a rotating sector $\left(r^{1}\right.$ shape for small-angle studies and $r^{2}$ for intermediate angles) before being recorded on photographic plates. During individual pulses the motion of the rotating sector is nearly frozen by the brief 0.2 to $1 \mathrm{~ms}$ electron shutter time and the individual contributions to the accumulated diffraction patterns are far from symmetric, axially. Integrated patterns, typically consisting of $10^{3}$ exposures registered on a plate, are satisfactorily averaged and not significantly degraded because the reciprocity law is well obeyed for exposures by fast electrons.

Pulsed operation makes it possible to monitor the spatial distribution of the effluent supersonic jet (skimmer removed) directly in the diffraction chamber with a fast ion gauge without interference by the substantial background gas that accumulates during operation in the continuous flow mode. Characteristic times for a gas pulse to pass a probing electron beam or ion gauge, rebound from the wall, and return to the probe are $\approx 0.6 \mathrm{~ms}$. Measurements of pulse densities are easily made before this interference can occur. Therefore, neither cryopumping nor fast diffusion pumps are required to keep effects of background gas minimal.

\section{Characteristic results}

In the absence of a skimmer, the spatial distribution of gas flowing from our standard Laval nozzle can be represented by a second-order Witch of Agnesi [2]. Profiles of cluster beams are nearly Gaussian and much narrower. Of special interest is the great fractionation between clusters and carrier gas implied by these profiles. Narrow cluster beams may be enriched, on axis, relative to initial concentrations in the carrier by factors of several hundred. It is this characteristic of nozzle flow that enables useful diffraction patterns to be taken under some conditions even when no skimmer is present.

As the initial pressure and/or molecular weight of monoatomic carrier gas are increased, the gas jet narrows as might be expected from considerations of inertial versus viscous effects. As the gas jet narrows, however, the cluster beam broadens by virtue of the increased scattering of clusters by collisions with gas molecules. The natural fractionation in micronozzles, then, is a strong function of pressure and carrier composition, and it depends on nozzle diameter and shape, as well.

Although the gas jets are broad compared with the cluster beams, they are narrow in comparison with those formed in free jet expansion. Time of flight measurements show that such concentrated jets as were commonly studied at Michigan in the past suffer a severe skimmer interaction. Clusters generated in flow through Laval nozzles tend to be so large, however, that they are able to plow through the nearly stagnant gas in the vicinity of the skimmer throat with little loss of velocity and, usually, only minor consequences. It is now possible to reduce such interactions drastically.

In prior research [3] we discovered that we can often alter the internal structures of clusters produced by varying flow conditions. In some cases clusters can be generated either in liquid or in solid form. In others, clusters can be induced to grow in two or even three different solid phases in pure form or mixed. Underlying principles are poorly understood. Our present experiments also show that high carrier pressures can produce clusters that are appreciably colder than clusters condensed from neat gas and cooled solely by evaporation.

Overall, the new pulsed system shows great promise in the study of clusters by electron diffraction. A 
much wider range of expansion conditions can be explored, and experiments are freer of deleterious interactions that complicate analyses of results. Results are expected to shed light on nucleation and mechanisms of nonequilibrium growth.

\section{References}

1. Bartell, L.S., Heenan, R.K., Nagashima, M.: J. Chem. Phys. 78, 234 (1983).

2. Bartell, L.S., Kacner, M.A., Goates, S.R.: J. Chem. Phys. 75, 2730 (1981)

3. Bartell, L.S., Harsanyi, L., Valente, E.J.: NATO ASI Ser., B. 158, $31(1987)$ 